\title{
DISTRIBUTION OF SPHAGNUM QUINQUEFARIUM IN THE CARPATHIAN BASIN ON THE BASIS OF LITERATURE AND HERBARIUM DATA
}

\author{
Tamás MISIK ${ }^{1}$, Dóra MISIK-BARTóK ${ }^{2}$, Sándor MOLJÁK ${ }^{3}$ \\ ${ }^{1}$ Eszterházy Károly University, Department of Environmental Science and Landscape Ecology, \\ Leányka str. 6-8, H-3300 Eger, Hungary \\ ${ }^{2}$ Government Office of Heves County, District Office of Eger, Public health, Kossuth Lajos str. 11, \\ H-3300 Eger, Hungary \\ ${ }^{3}$ Eszterházy Károly University, Innoregion Knowledge Centre, Leányka str. 6-8, H-3300 Eger, Hungary \\ e-mail: misik.tamas@uni-eszterhazy.hu
}

\begin{abstract}
We have found Sphagnum colonies on 10 October 2010 near Parádfürdő village, at the entrance of Ilona valley in northeast Hungary. The species was determined as Sphagnum quinquefarium (Lindb. ex Braithw.) Warnst. (five-ranked bog-moss), a protected species (on the basis of the 66/2015. (X. 26.) FM regulations) which was not until now described in the area of the Mátra Mountains. This species became known only from a few localities in Hungary, but was more frequent on the edge of the Carpathian Basin and in part of the outside border of our country. We collected the relevant data for the S. quinquefarium from the largest Herbarium database in Hungary and the countries of the Carpathian Basin and used the available botanical literature database. The peat moss species studied is very common in the High Tatras of Slovakia and throughout the Carpathian Mountains of Romania. This present paper summarises the recent distributional knowledge about S. quinquefarium distribution in the Carpathian Basin, outside of Hungary, on the basis of herbarium and literature data.
\end{abstract}

Keywords: peat moss, chorology, habitats, moorlands, herbarium data, literature data.

\section{Introduction}

We have found several Sphagnum colonies of different size on 10 October 2010 near Parádfürdö village in Hungary, at the entrance to the protected Ilona valley. The species was determined as Sphagnum quinquefarium (Lindb. ex Braithw.) Warnst. (five-ranked bog-moss) which was not until now known from the area of the Mátra Mountains (Misik and Misik-Bartók 2010, 2011). Our paper, which presents the distribution of the S. quinquefarium in the Carpathian Basin, in the neighbouring countries, focuses on this species.

Similar to the other inland peat mosses $S$. quinquefarium is protected, with an ecological value of 5000 HUF (66/2015. (X. 26.) FM regulations). The species studied is recognised in the Red List of Hungarian Bryophytes as having Vulnerable status (Papp et al. 2010), and as well in the recent version of the Red Data Book of European Bryophytes (Hodgetts 2015).

Peat mosses and their habitats are very rare in Hungary; this studied species was known up until now from only a few localities. It was first published from Hungary by Pócs (1958) in the Vend (Hungarian Slovenes) country near Szakonyfalu. In the 1960s it was recorded from the Vas ridge from Farkas-forest of Petömihályfa and also along the Kemence brooklet in NorthHungary (Boros 1968). On the basis of the studies in recent years researchers were able to find a 
small S. quinquefarium patch by Kishuta in Zemplén Mountains in North-eastern Hungary. The species is not common in western Hungary either, but is generally known from the area of Vend country, Örség, Kőszeg Mountains and Vas ridge (Szurdoki 2005). The volume of "Protected plant species of Hungary" (Farkas 1999) described only four assured habitats of this peat moss: (1) Zemplén Mountains (valley of the Kemence brooklet); (2) Kemeneshát (Petőmihályfa); (3) some points of Vend country and (4) Örség (Szarvaskend).

It occurs in many countries of Central and Western Europe. For example, the species is relatively common in the pre-Alpine zone of Alps at 450-1900 m a.s.1. in Switzerland (Feldmeyer-Christe et al. 2001). It is prevalent too in the boreal forests of Norway (Økland 1994) and has been found in the Netherlands (Dirkse et al. 1988). In the Faroe Islands at over $300 \mathrm{~m}$ a.s.l. five-ranked bog-moss colonies occur particularly (Lange 1984). The species is more frequent on the edge of the Carpathian Basin and in part of the outside border of Hungary. It is common in the North-west and North (High Tatra) Carpathians and the whole of the Romanian Carpathians.

The aim of this study is to summarize the herbarium and literature collections of the distribution of $S$. quinquefarium in the Carpathian Basin.

\section{Material and Methods}

S. quinquefarium is a medium-sized species, with capitula parrot-green, yellowish-green, or less commonly, largely or all deep red. It is typical of five-ranked bog-moss that scattered leaves stand in five lines along the stem; almost all fascicles have three spreading branches. The capitula of the moss have a wide triangular form, only $1 \mathrm{~mm}$ in length. It is a sterile species in Hungary. The moss occurs in acidophilous beech forests, on wet andesite rocks and in wet coniferous forests (Farkas 1999). Compared to other species in the Sphagnaceae family all fascicles of $S$. quinquefarium have 3 spreading branches, normally two in the other species. It is commonly confused with red bog-moss (Sphagnum capillifolium (Ehrh.) Hedw.), but that species usually has only two spreading branches per fascicle. This species has a flatter capitulum and stem leaves with the basal part parallel-sided (Atherton et al. 2010).

We collected the relatively scanty literature data on $S$. quinquefarium by means of the largest scientific database and searching programme. The relevant herbarium databases for the Carpathian Basin were collected from the Herbarium of Eszterházy Károly University in Eger (EGR), the Herbarium of Hungarian Natural History Museum (BP) in Budapest, Herbarium of the University of Debrecen (DE), Herbarium of the University of Pécs (JPU), Herbarium of the Alexandru Borza Botanical Garden (CL) at Babeş-Bolyai University of Cluj-Napoca, Herbarium of the Tatra National Park (TNP) in Tatranska Lomnica, Herbarium of the BZ Univerzity Komenského Bratislave in Pracovisko Blatnici (BBZ-K), personal Herbarium of D. Blanár (DB) and Herbarium of the Department of Botany in the Comenius University in Bratislava (SLO). The herbarium sheets were submitted for us and determined by Tamás Pócs and András Vojtkó from Eger, Erzsébet Szurdoki from Budapest, Attila Molnár V. from Debrecen, János Csiky from Pécs, Mihai Puşcaş, Irina Goia from Cluj-Napoca and Rudolf Šoltés, Katarína Mišíková in Slovakia. Relevant herbarium sheets for the peat moss were not recorded from the following important institutions: Herbarium of Eötvös Loránd University in Budapest, Herbarium of University of Sopron, Herbarium of Szent István University, Herbarium of Bakony Natural History Museum in Zirc, Móra Ferenc Museum of Szeged, Matra Museum of Gyöngyös, Savaria 
Museum of Szombathely, Herbarium of the Institute of Biology ZRC SAZU in Ljubljana, Herbarium of the Institute of Ecology of the Carpathians of NAS of Ukraine.

Sándor Molják (Innorégió Knowledge Centre of the Eszterházy Károly University) prepared a distributional map of the literature and herbarium data (Figure 1) using ArcGis software on the basis of the habitat's EOV coordinates.

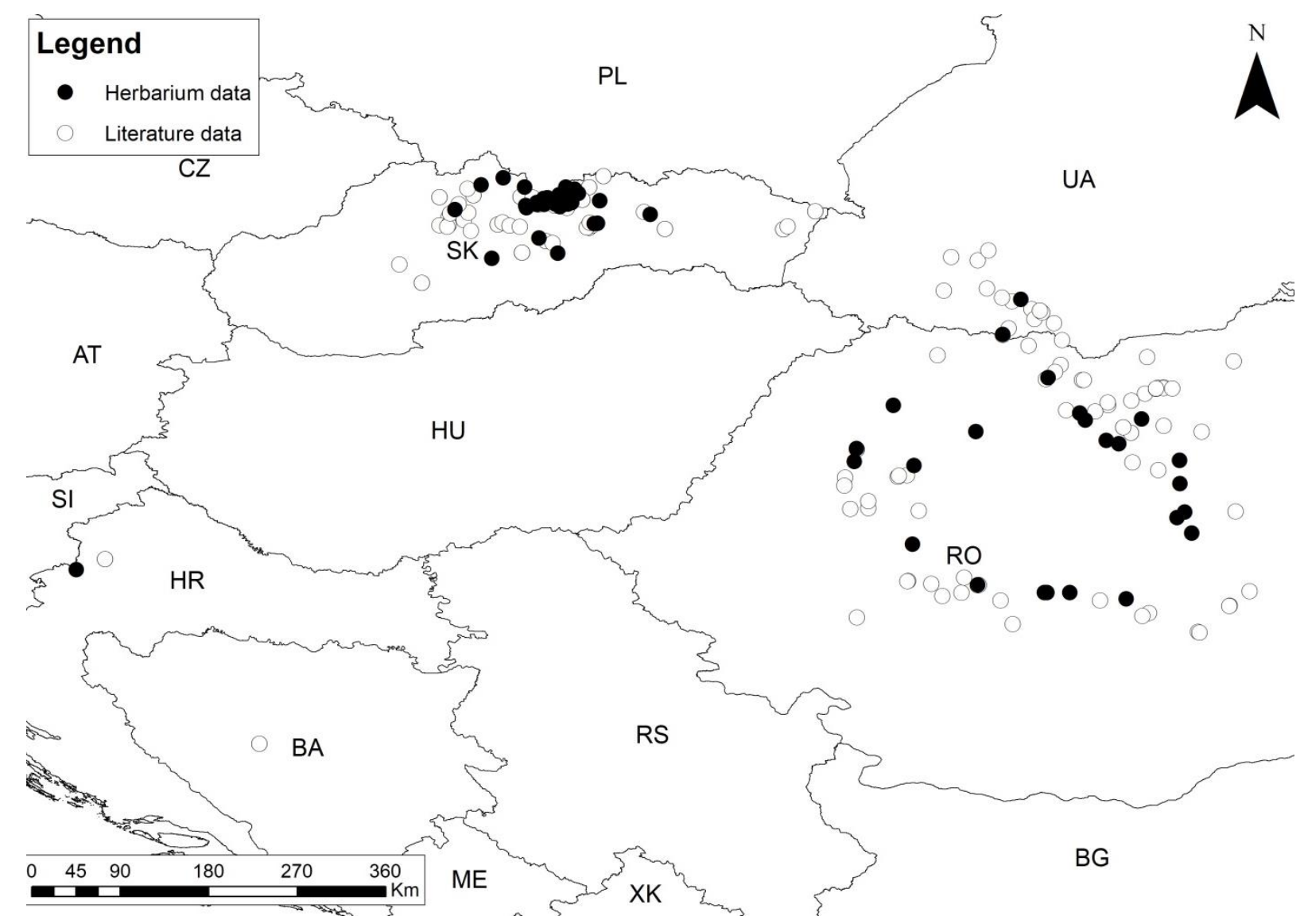

Fig. 1: Herbarium and literature data for the species Sphagnum quinquefarium in the Carpathian Basin (ed. Sándor Molják 2019)

\section{Results}

The peat moss studied is a frequent species with many habitats in Romania (i.a. Maramureș, Călimani and Făgăraș Mountains) Ukraine (Kárpátalja and Eastern Beskids Mountains) and Slovakia (i.a. High Tatra and Belanian Tatra Mountains); a few data are known from Czech Republic, outside of the Carpathian Basin. It cannot be found in Serbia, but at present the exploration of the potential moss habitats is underway (Sabovljević, pers. comm. 2018). It is known in Bosnia-Hercegovina from only one locality by Babin potok (on the basis of Sabovljević, pers. comm. 2018). In Croatia S. quinquefarium was found on granite and silicate rocks within beech woods in Blechno-Fagetum stands (Antun \& Vedran 2010). In Slovenia $S$. quinquefarium is a common peat-moss species, but only in the Julian Alps, Karavanks mountain range, Pohorje Mountains and Kamnik-Savinja Alps (Martinčič 2003, 2010, 2012; Kutnar \& Martinčič 2008; Kutnar 2013).

Romania - Literature data (mostly synthesized by Mohan 1998 and Plămadă 1998) are as follows (Figure 1): The species was found in the Maramureș Mountains: Pop Ivan Peak, Serban 
(Boros \& Vajda 1969), Tîjla Valley to Borșa Băi (Ștefureac 1958), Tăul Obcioarei (Goia et al. 2018); Rodnei (Radnai) Mountains: Pietrosul Peak, Piciorul Moşului, Ineu-Roșu Peak, Lala Lake (Ștefureac 1958); Bîrgăului Mountains: Grădinița peatbog (Silaghi \& Ștefureac 1969), Cucuresa Forest (Ștefureac et al. 1976); Călimani Mountains: peatbog near Fântânele Monastery (Ștefureac 1958), Poiana Ştampei peat bog (Ştefureac 1965, 1967), Drăgoiasa peatbog (Ştefureac 1962), Vatra Dornei (Breidler 1890; Papp 1934), Cristişor peatbog (Lungu 1973) by Poiana Puturosu (Büdös Tisztás) and by Jézer Lake (Erzberger et al. 2012); Obcina Mestecănișului Mountains: Lucina, Camionica Valley (Ștefureac 1958); Rarău (Ráró) Mountains: Codrul Secular Slătioara to "Latoace" stones and Ion Valley (Papp 1969; Ștefureac 1941, 1958), Pietrele Doamnei (Breidler 1890) Plaiul Todirescu (Ştefureac \& Raclaru 1978); Giumalău (Gyamaló) Mountains: Poiana Ițcani (Ștefureac 1958); Ceahlău Mountains (Papp 1934); Nemira (Nemere) Mountains: Slănic Valley (Papp 1957); Penteleu (Pintyiló) Mountains: Tisa Valley (Ștefureac 1958); Siriu Mountains: Buzăului Gorge, Lunca Băilor, Bocârnea Peak (Dihoru 1975), Giurgeului Depression (Lungu \& Raţiu 1975); Iezer-Păpusa (Jézer) Mountains (Mohan 1978); Bucegi (Bucsecs) Mountains by Lăptici (Ștefureac 1958); Cozia (Kozia) Mountains: Cozia Peak (Dihoru 1990); Lotrului Mountains: Repedea Valley (Ștefureac 1958; Ştefureac et al. 1959); Cindrel Mountains: Ciban (Borza 1959), Cotorești, Crinț, Găușoara, Iujbea, Săroi (Gündisch 1977), Păltiniș, Beșineu Mt. (Gündisch 1977; Röll 1903); Şureanu Mountains: Sebeș Valley (Ștefureac 1958; Borza 1959), Prigoana, Oașa, Șureanu Peak (Borza 1959); Retezat (Retyezát) Mountains: Gemenea Valley, Dosul Bârlei, Turcului Valley, Negru Lake, Șesele ridge (Plămadă 1975); Apuseni Mountains: Someșul Cald Gorges (Szamosbazár) (Goia \& Mătase 2001), Arieşul Mare basin (Goia and Schumacker 2002), Ponor, Cetățile Ponorului, Gabena Valley, Pîrcanul (Ștefureac 1958), Vidra, Găina Massive, Drăganului Valley (Péterfi 1908), Someşul Rece-Mtele Mare, Dobrinu (Reketó) (Pop 1960), Răcătău (Schedulae ad floram Romaniae Exsiccatam 1921).

Romania - Herbarium data are as follows (Figure 1): Călimani Mountains: Piatra Orban, Colibița (Kolibica) to Bistița Bîrgăului (Bogóbeszterce) (Jávorka 1942 BP), Stânceni (Gödemesterháza) (Vida 1955 BP), Dragoiasa (Vajda 1972 BP), Lunca Bradului (Demeter 1886 CL), Mureșenii Bârgăului (leg. Forstner, det. Ștefureac 1946 CL); Rodnei (Radnai) Mountains: Cororngiș (Korongyis) (Gürtler 1913 CL), Feketevíz (Vajda 1972 BP); Făgăraș Mountains (Fogarasi-Alps): Arpaşelul and Șerbota (Pócs 1955 EGR, 1955 EGR), Sîmbăta Valley (Pócs 1956 EGR); Cindrel Mountains: Păltiniş (Vajda 1968 EGR); Apuseni Mountains: Drăganului Valley (Nagysebes, Dregán valley) (Boros 1941 EGR, CL and DE), Vlădeasa - Zîrna valley (leg. Gergely, det. Plămadă, 1968 CL), Someșul Mic (Győrffy 1903 CL), Someșul Rece near Betlen Stone (Győrffy 1903 CL), Răcătău (Péterfi 1921 CL), Dobrin (Anon. CL), Măgura (Győrffy 1902 CL); Harghita Mountains: Sândominic (Csíkszentdomokos by Pásztorbükk) (Pócs 1955 EGR), Sîntimbru Băi (Büdösfürdő) and Sîncrăieni (Csíkszentkirály) (Vajda 1970 EGR, 1970 EGR); Bodoc Mountains: Ciomatu Massive - Tusnád: Kokojszás, Szent Anna lake (Vajda 1962 BP), Tusnád: Kokojszás, Szent Anna lake (Vajda 1965 BP) and Roşu valley (Péterfi 1918 CL).

Ukraine - Literature data are as follows (Figure 1): Kárpátalja: Berlebászka, Brusztura: Urja, Hoverla (Hóvár), Mala Hoverla, Pozyzewska, Terebesfejérpatak, Czarnahora (Csornahora) (Wilczek 1931, Kozij 1934, Szafran 1936), Bliznica, Kisgorgán, Mencsul, Pietros (Boros and Vajda 1969); Eastern Beskids Mountains, Ukrainian Carpathians Mountains: Breskul, Pop Iwan pol. (Wilczek 1931, Kozij 1934, Szafran 1936), Grofa (Pozynych and Savitska 2010), Prykarpattia, Zakarpattia Oblast, Svidovec, Chyvchyny (carpaty.net 2019). 
Ukraine - Herbarium data are as follows (Figure 1): Kárpátalja: Kőrösmező (Boros 1934 EGR), Trebusafejérpatak (Boros 1942 EGR).

Slovakia - Literature data are as follows (Figure 1): The species was detected in the High Tatra Mountains: Skalnaté pleso (Györffy 1909), Žabej Bielovodskej doline (Hadač et al. 1986); Poprad Basin: Kežmarské Žl'aby, Nová Polianka, Gerlacov, Tatranské Matliare (Pilous 1971), Belanský potok (Šoltés 2006); Low Tatra Mountains: Demänovská Dolina (Pilous 1971), Ludarov kar under Dumbier, Vyšna Boca and Branisko - Smrekovice, Stratenska hornatina Vel'ký Kysel' and Suchá Belá (Šmarda 1948), Korytnica (Degen et al. 1923), Vel'ký Sokol (Pilous 1971); Belanian Tatra Mountains: (Györffy 1909); Beskids Mountains: Oravské Beskydy (Pilous 1971), Bukovské vrchy (Herben et al. 1980); Slovak Ore Mountains: Slovenský raj (Šmarda 1948), Muránska planina (Peciar 1974), Muránska Vysočina, Klenovesky Vepor and Veporské vrchy (Pilous 1971), Vel'ka Stožka - Kl'ak hill (Herben and Soldán 1987); West Tatra Mountains: (Šmarda 1948), Spišská Magura (Šmarda 1952), Kamenistá dolina (Pilous 1971); Vel'ká Fatra Mountains: Malá Fatra (Šmarda 1952), Branisko (Pilous 1971), Selenec - Čertova brána (Pilous 1980), Tlstá (Uhlířová \& Bernátová 1986), Hal’amova kopa, Skalná, Smrekovica, Ostré brdo (Šoltés et al. 2004), Lubochňa (Jurko and Peciar 1963); Vihorlat-Gutin: Vihorlatské vrchy (Peciar 1970), Sninský Kameň (Pilous 1971); Orava Magura: Dolný Kubín (Uhlírová et al. 2015); The study of Peciar (1955) described the species in the Pieniny Mountains. On the basis of the Boros publication (1961) in Prešovský kraj Košická kotlina was assigned the habitat for the five-ranked bog-moss. Hlaváček (1957) transmitted the Štiavnické Mountains.

Slovakia - Herbarium data are as follows (Figure 1): High Tatra Mountains: Nagykorpalak (Vajda 1961 EGR), Kamzik \& Nagy-Tarpatak (Vajda 1962, 1962 BP), Bielovodská dolina (Sofron 1975 TNP), Malá Svištovka, Javorová dolina, Štrbské Pleso Furkotská dolina, Kriváň, Surovec, Machy, Uhliščatká (Šoltés 1977, 1983, 1984, 1987, 1987, 1987, 1997, 2004 TNP), Velická dolina, Žabia Bielovodská dolina (Šoltésová 1981, 1982 TNP), Kôprová dolina (Rajcová 1986 TNP), Dlhý les kežmarský and Tatranská Polianka (Viceníková 1991, 1992 TNP), Zelené pleso and Trojrohé pleso (Jiroušek 2016 person. herbarium); Vel'ká Fatra Mountains: Krackov, Lubochna (Vajda 1967 EGR és BP); Belanian Tatra Mountains: Skalné vráta (Šoltés 1981 TNP), Monkova dolina (Šoltés 1993 TNP, Kulová 1993 TNP); Podtatranská brázda Furrow: Podspády-Bor, Peciská (Šoltés 1989, 1992 TNP); West Tatra Mountains: Oravice Mountains - Árvai Magura and Jedlini-Trstena (Vajda 1968, 1971 BP), Krížna dolina (Moravčíková 1986 TNP), Tichá dolina, Osobitá (Šoltés 1988, 1992 TNP); Polana: Kluka (Janisová 1995 TNP); Slovak Ore Mountains: Chyžnianska rokl’a (Peciar 1972 SLO), Muránska planina (Blanár 1999 DB, Kochjarová 2000 BBZ-K); Branisko Mountains: Šindliar (Dostál 1986 SLO); Žilinský kraj region: Tatra Magna: Potocky, Pribilina (Vajda 1971 BP), Tatra Magna: Podbanské and Potocky prope Hotel Esperanto Pribilina (Vajda 1972, 1972 BP); Prešovský kraj region: Szepestamásfalva (Boros 1958 EGR), Káposztafalva, Roth-canyon (Boros 1961 EGR).

Croatia - Literature data are as follows (Figure 1): The species has some habitats in the Medvednica Mountains, near the capital city Zagreb (Sabovljević 2006 \& Sabovljević pers. comm. 2018). In the study of Pichler (1928) described in Samobor: Ludvić potok and Lipovečka Gradna as the moss habitats at 200-250 m a.s.1. In Požega-Slavonia county Ellis et al. (2016) and (2018) reported the species from the Papuk Mountains, situated in the mainly lowland area of NE Croatia in Fagus sylvatica L. - S. quinquefarium forest stand. In the other part of the 
Papuk Mountains this peat-moss was for the first time observed and described in 2015 (Alegro et al. 2015).

Croatia - Herbarium data are as follows (Figure 1): Zagreb county: Zagreb, Samobor (Boros 1966 EGR). Požega-Slavonia county: Papuk, Sokolina (Csiky 2015 JPU).

\section{Discussion}

On the basis of literature and relevant herbarium data $S$. quinquefarium has become known only from a few habitats in Hungary, but is more frequent on the edge of the Carpathian Basin and in part of the outside border of our country. This moss species, which typically prefers upland stands and well-drained spruce-forests, especially was prevalent in the range of the Carpathian Mountains. It is most common and frequent in the High Tatras of Slovakia and throughout the Carpathian Mountains of Romania. Farther south it is rarer; in Croatia it was reported only in couple of sites around the capital Zagreb, whereas in Serbia it has not yet been recorded, although researchers estimate that it may occur. In the Balkan Peninsula this peat moss will probably emerge in gaps revealed by precise habitat mapping.

Acknowledgements: First, thanks are due to Irina Goia of Babeş-Bolyai University, Cluj-Napoca for advice and, in the results section, herbarium and literature data from Romania. We especially thank János Csiky from the University of Pécs in Hungary, Rudolf Šoltés from the University of Žilina in Slovakia, Katarína Mišíková from Comenius University in Bratislava of Slovakia, Patrícia Durčanová and Tomáš Peterka from Masaryk University in Czech Republic, Mihai Puşcaş from the Alexandru Borza Botanical Garden, Cluj-Napoca in Romania, Marko S. Sabovljević from the University of Belgrade in Serbia and Vasyl' Sabadosh from Uzhhorod University in Ukraine, for a substantial number of literature and herbarium data and additions.

\section{REFERENCES}

1. Alegro, A., Šegota, V., Samardžic', I., 2015, Beech forest with peat moss in the Papuk Mt-unique in the vegetation of Croatia. In: 36th Meeting of Eastern Alpine and Dinaric Society for Vegetation Ecology (17-20 June 2015), Osijek. Book of Abstracts. Zagreb: Croatian Botanical Society: 13.

2. Antun, A., Vedran, S., 2010, Habitats of peat mosses (Sphagnum L.) in Croatia. In V. Ranđelović (ed.): Abstracts of the 10th Symposium on the flora of southeastern Serbia and neighbouring regions: 25-26.

3. Atherton, I., Bosanquet, D.S.S., Lawley, M., (eds.) 2010, Mosses and liverworts of Britain and Ireland: A Field Guide, Mark Lawley publishing.

4. Boros, Á., 1961, Moose der Moore im Tale des Monok-Baches bei Košice, Biológia, 5: 367-369.

5. Boros, Á., 1968, Bryogeographie und Bryoflora Ungarns, Akadémia Kiadó, Budapest.

6. Boros, Á., Vajda, L., 1969, Bryoflora Carpathorum septentrionali-orientaliorum, Revue Bryologique et Lichénologique, 36 (3-4): 397-450.

7. Borza, A., 1959, Flora şi vegetaţia văii Sebeşului, Editura Academiei R.P.R., Bucureşti.

8. Breidler, J., 1890, Beitrag zur Mossflora der Bukowina und Siebenbürgens, Österreichische Botanische Zeitschrift, 40 (4): 148-152.

9. Degen, Á., Gayer, J., Scheffer, J., 1923, Ungarische Moorstudien I. Magyar Botanikai Lapok, 22: 1-116.

10. Dihoru, G., 1975, Invelişul vegetal din Muntele Siriu, Edit. Acad. R.S.R., Bucureşti.

11. Dihoru, G., 1990, Bryophytes of Cozia Mountains, Analele Universității București, Seria Biologie, 39: 51-59.

12. Dirkse, G.M., van Melick, H.M.H., Touw, A., 1988, Checklist of Dutch bryophytes, Lindbergia, 14: 167-175.

13. Ellis, L.T., Aleffi, M., Alegro, A., Segota, V., Asthana, A.K., Gupta, R., Singh, V.J., Bakalin, V.A., BednarekOchyra, H., Cykowska-Marzencka, B., Benitez, A., Borovichev, E.A., Vilnet, A.A., Konstantinova, N.A., Buck, W.R., Cacciatoro, C., Sérgio, C., Csiky, J., Deme, J., Kovács, D., Damsholt, K., Enroth, J., Erzberger, P., Fedosov, V.E., Fuertes, E., Gradstein, S.R., Gremmen, N.J.M., Hallingbäck, T., Jukonienè, I., Kiebacher, T., 
Larraín, J., Lebouvier, M., Lüth, M., Mamontov, Yu.S., Potemkin, A.D., Nemeth, Cs., Nieuwkoop, J.A.W., Nobis, M., Węgrzyn, M., Wietrzyk, P., Osorio, F., Parnikoza, I., Virchenko, V.M., Peralta, D.F., Carmo, D.M., Plášek, V., Skoupá, Z., Poponessi, S., Venanzoni, R., Puche, F., Purger, D., Reeb, C., Rios, R., RodriguezQuiel, E., Arrocha, C., Sabovljević, M.S., Nikolić, N., Sabovljević, A.D., dos Santos, E.L., Segarra-Moragues, J.G., Ştefănuţ, S., Stončius, D., 2016, New national and regional bryophyte records, 48, Journal of Bryology, 38 (3): 235-259. DOI: 10.1080/03736687.2016.1206685।

14. Ellis, L.T., Afonina, O.M., Andriamiarisoa, R.L., Asthana, G., Bharti, R., Aymerich, P., Bambe, B., Boiko, M., Brugués, M., Ruiz, E., Sáez, L., Cano, M.J., Ros, R., C`ihal, L., Deme, J., Csiky, J., Dihoru, G., Dřevojan, P., Ezer, T., Fedosov, V.E., Ignatova, E.A., Seregin, A.P., Garcia, C.A., Martins, A., Sérgio, C., Sim-Sim, M., Rodrigues, A.S.B., Gradstein, S.R., Reeb, C., Irmah, A., Suleiman, M., Koponen, T., Kučera, J., Lebouvier, M., LiQun, Y., Long, D.G., Maksimov, A.I., Maksimova, T.A., Muñoz, J., Nobis, M., Nowak, A., Ochyra, R., O’Leary, S.V., Osorio, F., Pisarenko, O.Yu., Plášek, V., Skoupá, Z., Schäfer-Verwimp, A., Schnyder, N., Shevock, J.R., Ștefănuț, S., Sulayman, M., Sun, B.-Y., Park, S.J., Tubanova, D.Ya., † Vánă, J., Wolski, G.J., Yao, K.-Y., Yoon, Y-J., Yücel, E., 2018 New national and regional bryophyte records, 56, Journal of Bryology, 40 (3): 271-296. DOI: 10.1080/03736687.2018.1487687

15. Erzberger, P., Höhn, M., Pócs, T., 2012, Contribution to the bryoflora of Călimani mountains in the Eastern Carpathians, Romania, I, Acta Biologica Plantarum Agriensis, 2: 75-97.

16. Farkas, S., (szerk.), 1999, Magyarország védett növényei, Mezőgazda Kiadó, Budapest.

17. Feldmeyer-Christe, E., Schnyder, N., Bisang, I., 2001, Distributions and habitats of peat mosses, Sphagnum, in Switzerland, Lindbergia, 26: 8-22. DOI: 10.2307/20150056

18. Goia, I., Mătase, D., 2001, Bryofloristical research in the Someşul Cald Gorges, Contrib.Bot., 36: 15-24.

19. Goia, I., Schumacker, R., 2002, Researches on the bryophytes from rotten wood in the Arieşul Mare basin, Contrib.Bot., 37: 85-94.

20. Goia, I., Şuteu, A., Țifrea, E., Grapini S., 2018, Studies on plant communities in Tăul Obcioarei peat bog Maramureş Mountains, Contrib.Bot., 53: 35-48.

21. Gündisch, F., 1977, Beitrag zu einer Moosflora des Zibin-Gebirges, Studii şi comunicări, Ştiinţe naturale, Muz. Brukenthal, 21: 43-77.

22. Györffy, I., 1909, Delphinium oxysepalum Borb. et Pax, Magyar Botanikai Lapok, 8: 337.

23. Hadač, E., Andresová, J., Paukertová, J., Klescht, V., 1986, Čtyři mokřadní rostlinná spoločenstva Bukovských vrchů na SZ Slovenska, Preslia, 58: 339-347.

24. Herben, T.J., Soldán, Z., Váňa J., 1980, Materiály k bryoflóre širšího okolí Sniny, Zpravodaj české Botanické společnosti, 15: 66-72.

25. Herben, T., Soldán, Z., 1987, Bryofloristic material from the central part of Muránska planina (Western Carpathians), Preslia, 59: 65-85.

26. Hlaváček, A., 1957, Výskyt a rozšírenie fialky močiarnej (Viola palustris L.) na Slovensku, Biológia, 12: 414420.

27. Hodgetts, N.G., 2015, Checklist and country status of European bryophytes - towards a new Red List for Europe, Irish Wildlife Manuals, No. 84. National Parks and Wildlife Service, Department of Arts, Heritage and the Gaeltacht, Ireland.

28. Jurko, A., Peciar, V., 1963, Pflanzengesellschaften an schattigen Felsen in den Westkarpaten, Vegetatio, 11: 199-209.

29. Kutnar, L., 2013, Visokobarjanska vegetacija v Sloveniji, Združbe šotnih mahov, rušja in smreke, Ljubljana.

30. Kutnar, L., Martinčič, A., 2008, Bryophyte species diversity of forest ecosystems in Slovenia (Intensive Monitoring Programme), Zbornik gozdarstva in lesarstva, 85: 11-26.

31. Lange, B., 1984, Sphagnum in Greenland, Svalbard, Iceland, and the Faroes, Lindbergia, 10: 133-158.

32. Lungu, L., 1973, Analiza brioflorei din lunca Borcutului de la Cristişor-Neagra Broştenilor (Carpaţii Orientali), Analele Universităţii Bucureşti. Seria Biologie Vegetală, 22: 69-76.

33. Lungu, L., Raţiu, F., 1975, Contribuţii la cunoaşterea brioflorei mlaştinilor eutrofe din depresiunea Giurgeului, Contrib.Bot., 15: 49-54.

34. Martinčič, A., 2003, Seznam listnatih mahov (Bryopsida) Slovenije, Hacquetia, 2 (1): 91-166.

35. Martinčič, A., 2010, Mahovna flora fitogeografskega podobmočja Dravski Kozjak (Slovenija), Hladnikia, 25: 13-30.

36. Martinčič, A., 2012, Mahovna flora fitogeografskega podobmočja Mežiško-Mislinjska dolina - Strojna 
(Slovenija), Hladnikia, 30: 45-58.

37. Misik, T., Misik-Bartók, D., 2010, Új tőzegmoha-előfordulás a Mátrában, Kitaibelia, 15: 180.

38. Misik, T., Misik-Bartók, D., 2011, Distribution of Sphagnum quinquefarium in Hungary, Acta Biologica Plantarum Agriensis, 2: 97-99.

39. Mohan, G., 1978, Morphogenetische, zytogenetische, ökologische und phytosoziologische Untersuchungen über die Bryophyten des Ieser-Päpuşa-Massivs, Feddes Repert., 89: 327-344.

40. Mohan, G., 1998, Catalogul Briofitelor din România, Acta Botanica Horti Bucurestiensis, Lucrările Grădinii Botanice, Editura Universitătii din Bucureşti.

41. Økland, R.H., 1994, Bryophyte and lichen persistence patterns in a Norwegian boreal coniferous forest, Lindbergia, 19: 50-62.

42. Papp, B., Erzberger, P., Ódor, P., Hoch, Zs., Szövényi, P., Szurdoki, E., Tóth, Z., 2010, Updated checklist and red list of Hungarian Bryophytes, Studia Botanica Hungarica, 41: 31-59.

43. Papp, C., 1934, Contribuţiune la bryogeografia Masivului Ceahlău, Buletinul Societăţii Regale Române de geografie, 52: 62-89.

44. Papp, C., 1957, Contribuţiuni la cunoaşterea florei şi vegetaţiei, în special a celei archegoniate, dintre văile rîurilor Oituz şi Uz, din regiunea Bacău, Analele ştiinţifice ale Universităţii "Al.I. Cuza" din Iaşi (serie nouă). Secţiunea II (Stiinţe Naturale-Geografie), 3(1-2): 279-310.

45. Papp, C., 1969, Contribuţiuni la cunoaşterea brioflorei Masivului Rarău din Carpaţii Răsăriteni, Analele ştiințifice ale Universităţii "Al.I. Cuza" din Iaşi (serie nouă), Secţiunea II. a. Biologie, 15 (1): 187-193.

46. Peciar, V., 1955, Machorasty (Bryophyta) slovenskej časti Pienin. Pr. II. Sekcie Slov. Akad. Vied., Ser. Biologica, 1: 1-29.

47. Peciar, V., 1970, Studia bryofloristica Slovaciae II, Acta Fac. Rerum Nat. Univ. Comenianae, ser. Bot., 16: 2735.

48. Peciar, V., 1974, Studia bryfloristica Slovaciae VI, Acta Fac. Rerum Nat. Univ. Comenianae, ser. Bot., 22: 93101.

49. Péterfi, M., 1908, Adatok a Biharhegység Mohaflórajanak Ismeretehez, Math. Termeszet. Közlemények, 3: 261332.

50. Pichler, A.R., 1928, Mahovi tresctari Hrvatske i Slovenije, Acta Botanica Croatica, 3: 41-60.

51. Pilous, Z., 1971, Bryophyta - Mechyorosty. Sphagnidae - Mechy rašeliníkové, Nakladatelství Československá Akademie Vêd, Praha.

52. Pilous, Z., 1980, Mechorosty Blatnické doliny a Gaderské doliny ve Velké Fatře, Výsk. Práce Ochr. Prír., Bratislava, 3B: 111-140.

53. Plămadă, E., 1975, Flora și vegetația briologică din rezervația ştiinţifică a Parcului Național Retezat, Teza de doctorat. Univ. Bucureți.

54. Plămadă, E., Dumitru, C., 1998, Flora briologică a României, Clasa Musci. Vol. I. Fasc. I. Sphagnales Andreaeales - Tetraphidales - Buxbaumiales - Schistostegales - Polytrichales - Fissidentales - Archidiales Seligeriales, Presa Universitară Clujeană, Cluj-Napoca.

55. Pop, E., 1960, Mlaștinile de turbă din Republica Populară Româna, Ed. Acad. R.P.R.

56. Pozynych, I.S., Savitska, A.G., 2010, Characteristics of vascular and Bryophyte flora in the Landscape Reserve Grofa (The Ukrainian Carpathians), Visnyk of Dnipropetrovsk University. Biology. Ecology, 18: 69-75.

57. Pócs, T., 1958, Beiträge zur Moosflora Ungarns und der Ost- und Südkarpaten, Annales Historico-Naturales Musei Nationalis Hungarici, 50: 107-119.

58. Röll, J., 1903, Beiträge zur Moosflora der Transsilvanischen Alpen, Hedwigia, 42 (6): 297-305.

59. Sabovljević, M., 2006, Checklist of mosses of Croatia, Archives of Biological Science Belgrade, 58: 45-53. DOI: $10.2298 / \mathrm{ABS} 0601045 \mathrm{~S}$

60. Silaghi, G., Ștefureac, T., 1969, Cateva macromicete din turbării noi pentru Romania, Contrib.Bot., 9: 89-95.

61. Šmarda, J., 1948, Mechy Slovenska, Čas. Zem. Museum Brno, 32: 1-75.

62. Šmarda, J., 1952. První doplněk k Mechům Slovenska, Čas. Mor. Museum, 37: 26-31.

63. Šoltés, R., 2006, Príspevok k bryoflóre mokradí k. Ú. Východná (Liptovská Kotlina, Slovensko), Naturae Tutela, 10: 5-9.

64. Šoltés, R., Kubinská, A., Kliment, J., Bernátová, D., 2004, Machorasty Vel'kej Fatry, Ochr. Prír., Banská Bystrica, 23: 58-95.

65. Ştefureac, T.I., 1941, Synecological and sociological researches on Bryophytes from Codrul Secular Slătioara 
(Bucovina), Analele Acad. Române, Mem. Sect. Şti., Ser. III, 16: 1133-1329.

66. Ştefureac, T.I., 1958, Contribuţii la cunoaşterea şi răspândirea speciilor genului Sphagnum L. in bryoflora ţării, Contrib.Bot., 1: 91-115.

67. Ştefureac, T.I., 1962, Relictes subartiques dans la bryoflore du marais eutrophe de Drăgoiasa Carpathes Orientales, Revue Bryologique et Lichénologique, 31 (1-2): 68-73.

68. Ştefureac, T.I., 1965, Rezervaţia Tinovul Mare de la Poiana Stampei (Căsoi). In: Ghid geobotanic pentru Moldova de Nord, Consfăt. a V-a de Suceava: 39-47.

69. Ştefureac, T.I., 1967, Relicte arctice şi subarctice în brioflora Carpaţilor sud-estici, Lucr. Grăd. Bot. Bucureşti, Acta Bot. Horti Bucurestiensis, 1966: 305-324.

70. Ştefureac, T.I, Raclaru, P., 1978, Consideraţii asupra caracterului florei din Rezervaţia Plaiul Todirescu Slătioara (Bucovina), Lucrările Grădinii Botanice din București, Acta Botanica Horti Bucurestiensis, 19771978: 191-208.

71. Ştefureac, T.I., Popescu, A., Lungu, L., 1959, Noi contribuţii la cunoaşterea florei şi vegetaţiei Bryophytelor din Valea Lotrului, Stud. Cercet. Biol., Ser. Biol. Veg., 11 (1): 7-61.

72. Ștefureac, T.I., Mihai, G., Pascal, P., 1976, Cercetäri briologice in rezervatia forestierä Cucureasa (Vatra Dornei), St. și Cerc. Biol., Ser. Biol. veg., 28 (1): 35-42.

73. Szurdoki, E., 2005, Magyarországi tözegmohafajok elterjedése és egyes fajok vizkémiai igényének vizsgálata, Doktori értekezés, ELTE.

74. Uhlíŕová, J., Bernátová, D., 1986, Nové lokality bradáčika srdcovitého [Listera cordata (L.)Br.] vo Vel'kej Fatra a v Slovenskom raji, Biológia, 41: 495-497.

75. Uhlíŕová, J., Bernátová, D., Šibík, J., 2015, Fenomén vrchoviskových komplexov Hornej Oravy, Acta Rerum Naturalium Musei Nationalis Slovaci, 61: 34-65.

76. 66/2015. (X. 26.) FM rendelet az elkobzott védett természeti értékekkel kapcsolatos intézkedésekről szóló 19/1997. (VII. 4.) KTM rendelet, valamint a védett és a fokozottan védett növény- és állatfajokról, a fokozottan védett barlangok köréről, valamint az Európai Közösségben természetvédelmi szempontból jelentős növény- és állatfajok közzétételéről szóló 13/2001. (V. 9.) KöM rendelet módosításáról.

\section{DISTRIBUȚIA SPECIEI SPHAGNUM QUINQUEFARIUM ÎN BAZINUL CARPATIC PE BAZA DATELOR DIN LITERATURĂ ŞI DIN HERBARE}

\section{(Rezumat)}

S-au găsit colonii de Sphagnum în data de 10 octombrie 2010 lângă localitatea Parádfürdő, la intrarea de pe valea Ilona, în nord-estul Ungariei. Specia a fost determinată ca Sphagnum quinquefarium (Lindb. ex Braithw.) Warnst., o specie protejată (pe baza legii FM 66/2015 (X. 26.)), care nu a mai fost descrisă din zona Munţilor Mátra. Specia se cunoştea doar din câteva localităţi din Ungaria, dar era mai frecventă la limita bazinului carpatic şi parţial înafara graniţelor ţării noastre. Datele relevante pentru S. quinquefarium au fost colectate din cea mai mare bază de date de herbar din Ungaria şi ţările bazinului carpatic şi din bazele de date botanice din literatura disponibilă. Specia studiată este foarte comună în zona înaltă a munţilor Tatra din Slovacia şi în Munţii Carpaţi din România. Acest studiu arată distribuţia actuală a speciei $S$. quinquefarium în bazinul carpatic, înafara Ungariei, pe baza datelor de herbar şi din literatură. 